\title{
Complexity of the Fibonacci snowflake
}

\author{
A. BLONDIN MASSÉ, S. BRLEK ${ }^{\dagger}$, S.LABBÉ, AND M. MENDÈS FRANCE
}

to la mémoire de Benoit ctoandelbrot

\begin{abstract}
ctbstract. Che object under study is a particular closed curve on the square lattice $\mathbb{Z}^{2}$ related with the Fibonacci sequence $F_{n}$. It belongs to a class of curves whose length is $4 F_{3 n+1}$, and whose interiors by translation tile the plane. The limit object, when conveniently normalized, is a fractal line for which we compute first the fractal dimension, and then give a complexity measure.
\end{abstract}

\$1. Introduction. In a recent article [1] we defined and studied the properties of what we named a Fibonacci snouflake. Let us recall the main facts. Consider the infinite sequence $\left(q_{n}\right)_{n \in \mathbb{N}}$ defined recursively on the alphabet $\mathcal{T}=\{L=$ left, $R=$ right $\}$ by

$$
\begin{aligned}
& q_{0}=\varepsilon \text { (the empty word), } q_{1}=R, \\
& q_{n}= \begin{cases}q_{n-1} q_{n-2} & \text { if } n \equiv 2 \bmod 3, \\
q_{n-1} \overline{q_{n-2}} & \text { if } n \equiv 0,1 \bmod 3 .\end{cases}
\end{aligned}
$$

Here, $\overline{q_{n-2}}$ represents the word $q_{n-2}$ in which the letters $R$ and $L$ are permuted. The length $\left|q_{n}\right|$ of the word $q_{n}$ satisfies the Gibonacci relation

$$
\left|q_{n}\right|=\left|q_{n-1}\right|+\left|q_{n-2}\right|
$$

and therefore

$$
\left|q_{n}\right|=\frac{1}{\sqrt{5}}\left(\frac{1+\sqrt{5}}{2}\right)^{n}-\frac{1}{\sqrt{5}}\left(\frac{1-\sqrt{5}}{2}\right)^{n} .
$$

Fo each word $w \in \mathcal{T}^{*} \cup \mathcal{T}^{\mathbb{N}}$ corresponds a polygonal line $\Pi$ on the lattice $\mathbb{Z}^{2}$. otot the $n$-th vertex of $\Pi$, the $n$-th letter $w_{n}$ of $w$ indicates the direction of the next side. It may well happen that some sides are visited several times as for the case $w=L^{4}$ for example. The length of

+ With the support of NSERC (Canada). 
the polygonal line $\Pi$ corresponding to $w=L^{4}$ is: $|\Pi|=\left|L^{4}\right|+1$. Hctually, the following general property holds

$$
|\Pi|=|w|+1, \forall w \in \mathcal{T}^{*} .
$$

to finite polygonal line on $\mathbb{Z}^{2}$ is closed if both extremities coincide. The corresponding word is then said to be closed. Open signifies nonclosed; for example, $L^{4}$ is open while $L^{3}$ is closed. On open polygonal line is non-intersecting if each of its vertices is attained once only. do closed non-intersecting polygonal line is one for which the only vertices visited twice are the extremities. Here are a ferv examples:

(i) $L^{4}$ is neither closed nor non-intersecting;

(ii) $L^{3}$ is closed and non-intersecting;

(iii) $(L R)^{n}$ is open and non-intersecting;

(iv) $L^{3} R L^{3}$ is closed but not non-intersecting;

(v) $(L R L)^{3} L R$ is closed and non-intersecting.

Given a word $w \neq \varepsilon$, we denote $w^{-}$the word where the last letter is suppressed. For example, $(L R L)^{4^{-}}$is closed and non-intersecting. In [1] we showed that the polygonal line $\Pi_{n}$ corresponding to the word $\left(q_{3 n+1}\right)^{4^{-}}$is closed and non-intersecting (Theorem 10) (see Figure 1 ). Therefore, the property (1) implies that

$$
\left|\Pi_{n}\right|=4\left|q_{3 n+1}\right|
$$

that is, the polygon $\Pi_{n}$ is composed of $4\left|q_{3 n+1}\right|$ unit segments in $\mathbb{Z}^{2}$.

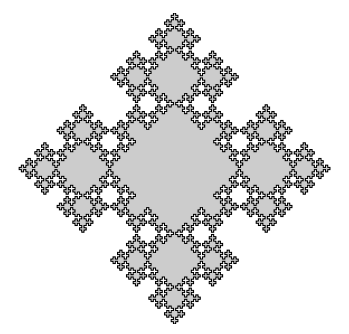

Figure 1. Fibonacci snowflake of order $n=5$. 
\$2. Fractal dimension. "The rather "complicated" associated polygon $\Pi_{n}$, when conveniently normalized, tends to a fractal line as $n \rightarrow \infty$ which we call Tibonacci snowflake. In our previous article we were aware of its complexity, yet we overlooked its fractal dimension even though we had all the information needed. We now prove:

Theorem 1. The fractal dimension of the Tibonacci snouflake is

$$
d=\frac{\log (2+\sqrt{5})}{\log (1+\sqrt{2})}=1.637938210 \cdots
$$

Proof. First of all, we must see how to normalize the sequence of polygons $\Pi_{n}$ so that they stary in a bounded region of the plane as $n \rightarrow \infty$. In our previous article we observed that the smallest square with sides parallel to the two axes $O x, O y$ and containing $\Pi_{n}$, has sides of size $2 P(n+1)-1$ where the recurrence

$$
P(0)=0, P(1)=1 ; P(n)=2 P(n-1)+P(n-2) \text {, for } n>1,
$$

defines the so-called Pell numbers. Then they satisfy the relations

$$
\begin{aligned}
P(n+1) & =\frac{2+\sqrt{2}}{4}(1+\sqrt{2})^{n}+\frac{2-\sqrt{2}}{4}(1-\sqrt{2})^{n} \\
2 P(n+1)-1 & =\frac{2+\sqrt{2}}{2}(1+\sqrt{2})^{n}+\frac{2-\sqrt{2}}{2}(1-\sqrt{2})^{n}-1 \\
& \sim \frac{2+\sqrt{2}}{2}(1+\sqrt{2})^{n} .
\end{aligned}
$$

The polygon $\Pi_{n}$ is composed of $4\left|q_{3 n+1}\right|$ unit segments and blows up as $n \rightarrow \infty$. But the normalized polygon $\frac{1}{2 P(n+1)-1} \Pi_{n}$ stays bounded. It has $4\left|q_{3 n+1}\right|$ sides each of length $(2 P(n+1)-1)^{-1}$. Che total d-dimensional normalized polygon has length

$$
\frac{4\left|q_{3 n+1}\right|}{(2 P(n+1)-1)^{d}}
$$

and therefore the fractal dimension of the Fibonacci snowflake is

$$
d=\lim _{n \rightarrow \infty} \frac{\log \left(\left|q_{3 n+1}\right|\right)}{\log (P(n+1))}=\lim _{n \rightarrow \infty} \frac{\log \left(\frac{1+\sqrt{5}}{2}\right)^{3 n+1}}{\log (1+\sqrt{2})^{n}}=\frac{\log (2+\sqrt{5})}{\log (1+\sqrt{2})} .
$$


\$3. A measure of complexity. There are obviously many ways to measure the complescity of a line. We mention below a measure related to the number of points of intersection of the figure with a random straight line.

Recall a classical result due to Cauchy [2], Crofton [3], Steinhaus [5]. Consider a plane rectifiable curve $\Gamma$ of length $|\Gamma|$ and whose convex hull $K$ has a frontier of length $|\partial K|$. to straight line $D$ is defined by

$$
x \cos \theta+y \sin \theta-\rho=0 .
$$

The probability measure considered is the uniform Lebesgue measure $d \rho . d \theta$ conditioned by the fact that $D$ intersects $\Gamma$. The result we alluded to is that the average number of intersecting points of $\Gamma$ with a random $D$ is

$$
N=\frac{2|\Gamma|}{|\partial K|}
$$

opplying this to $\Pi_{n}$, we see that the average number $N_{n}$ of intersection points of $\Pi_{n}$ with $D$ is

$$
N_{n}=2 \frac{2\left|q_{3 n+1}\right|}{\left|\partial K_{n}\right|}
$$

where $K_{n}$ is the convex hull of $\Pi_{n}$. Easily seen, $\left|\partial K_{n}\right|$ is of the order of $P(n+1)$. Therefore,

Theorem 2. We have

$$
N_{n} \sim a \frac{(2+\sqrt{5})^{n}}{(1+\sqrt{2})^{n}}=a\left(\frac{2+\sqrt{5}}{1+\sqrt{2}}\right)^{n} .
$$

There is no difficulty to compute the constant $a=(1+\sqrt{5})$, but the important point is that the average number $N_{n}$ of intersection points increases exponentially, showing once more the high complexity of the Fibonacci snowflake. Theorem 2 could obviously be considered as a corollary of the proof of Theorem 1. Both results are strongly related. 
Remark 1. Let $\delta_{n}$ be the diameter of $\Pi_{n}$ (which is of the order of $P(n+1)$ ). Obviously, $2 \delta_{n} \leq\left|\partial K_{n}\right| \leq \pi \delta_{n}$. Cheorem 2 states that the ratio $\mathcal{L}_{n} / \delta_{n}$ is of the order of $N_{n}$ where $\mathcal{L}_{n}$ is the length of $\Pi_{n}$. Che difference

$$
\mathcal{L}_{n}-\delta_{n}=\delta_{n}\left(\frac{\mathcal{L}_{n}}{\delta_{n}}-1\right)
$$

measures the distance of $\Pi_{n}$ from being a straight line. Che larger the ratio, more the curve meanders.

Remark 2. Let $F$ be a positive strictly increasing function. The ratio $F\left(\mathcal{L}_{n}\right) / F\left(\delta_{n}\right)$ is a measure of the complexity of $\Pi_{n}$. When $F=\log$ we obtain Cheorem 11 and when $F=I_{d}$ we obtain Cheorem 2. The choice $F=\exp$ leads to $\exp \left(\mathcal{L}_{n}-\delta_{n}\right)$ i.e. Remark 1 .

\$4. Entropy. Let $p_{j}^{(n)}$ be the probability that a random straight line intersects $\Pi_{n}$ in exactly $j$ points, given that the line meets $\Pi_{n}$. The associated entropy $h_{n}$ is by definition

$$
h_{n}=-\sum p_{j}(n) \log \left(p_{j}(n)\right) .
$$

In [4] it is shown that

$$
h_{n} \leq \log \left(\frac{2 \mathcal{L}_{n}}{\left|\partial K_{n}\right|}\right)+\left(1-\frac{\left|\partial K_{n}\right|}{2 \mathcal{L}_{n}}\right) \log \left(\frac{2 \mathcal{L}_{n}}{2 \mathcal{L}_{n}-\left|\partial K_{n}\right|}\right) .
$$

The second term in the righthand side is positive, less than 1 and tends to 0 with $\frac{\left|\partial K_{n}\right|}{\mathcal{L}_{n}}$.

Since $\mathcal{L}_{n}$ is of the order of $(2+\sqrt{5})^{n}$ and $\left|\partial K_{n}\right|$ is of the order of $(1+\sqrt{2})^{n}$, we have

$$
h_{n} \leq n \log \left(\frac{2+\sqrt{5}}{1+\sqrt{2}}\right)+\mathcal{O}(1) .
$$

We have thus established an upper bound for the complexity of $\Pi_{n}$ :

Theorem 3.

$$
\limsup _{n \rightarrow \infty} \frac{1}{n} h_{n} \leq \log \left(\frac{2+\sqrt{5}}{1+\sqrt{2}}\right)
$$




\section{References}

[1] to. Blondin. Massé, Y. Brilek, Y. Labbé, and Mo. Hendès France, Fibonacci snowflakes, donn. Sci. doath. Québec 35 (2011), to appear.

[2] do.L. Cauchy, thémoire sur la rectification des courbes et la quadrature des surfaces courbes, doém. Atocad. Sci. 22 (1850), 3-15.

[3] MO. U9. Crofton, On the theory of local probability, applied to straight lines drawn at random in a plane; the methods used being also extended to the proof of certain new theorems in the integral calculus, Thil. Crans. of the royal soc. London 158 (1868), 181-199.

[4] Mo. Wendès France, The Planck constant of a curve, in Fractal Geometry and tonalysis (7. Bélair and Y. Dubuc, eds.), vol. 346. Thluver tocademic Publishers, 1991, pp. 325-366.

[5] H. Steinhaus, Length, shape and area, Collog. Moath. 3 (1954), 1-13.

to. Blondin Massé, Y. Brilek, Y. Labbé, LaCIM, Université du Québec à Montréal,

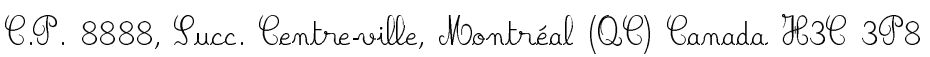

E-mail address: \{blondin_masse.alexandre, labbe.sebastien\}@courrier.uqam.ca,

brlek.srecko@uqam.ca

Mo. Mendès France, Département de mathématiques, Uollog 5251, Université Bordeaux 1,351 cours de la Libération., F.33405 Calence cedex, France

$E$-mail address: miche1.mendes-france@math.u-bordeaux1.fr 